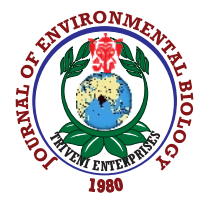

\title{
External and internal morphology, and feeding ability of yellow seahorse Hippocampus kuda (Bleeker, 1852) early juveniles
}

\author{
Mohd. S. Kamarudin ${ }^{1,2}$, N. Romano ${ }^{3}$, A. Arshad ${ }^{1,2}$, N.L.W.S. Wong ${ }^{1,2}$ and J.C. Teh ${ }^{1 *}$ \\ ${ }^{1}$ Department of Aquaculture, Faculty of Agriculture, Universiti Putra Malaysia, 43400 Serdang, Selangor, Malaysia \\ ${ }^{2}$ International Institute of Aquaculture and Aquatic Sciences, Universiti Putra Malaysia, 71050 Teluk Kemang, Port Dickson, Malaysia \\ ${ }^{3}$ Aquaculture/Fisheries Center, University of Arkansas at Pine Bluff, 1200 North University Drive, Pine Bluff, AR 71601, USA \\ *Corresponding Author Email : jun_chin@yahoo.com
}

\section{Abstract}

Aim: Despite their well-known strategy of brooding in male pouches, survival of early juveniles of seahorses still represents a significant bottleneck to its population survival. This study aimed to address the feeding and digestive efficiency of early Hippocampus kuda in order to improve their survival.

Methodology: The external and gut morphology of $H$. kuda juveniles in the immediate period after being released from the brood pouch ( $0-9$ day-afterbirth) were examined and described through histological and microscopic methods.

Results: Unlike premature seahorses with a round-shaped yolk sac attached to their semi-transparent body, the normal newborns had no external yolk sac but some yolk residues were observed internally. Although the mouth gape size, mouth area, height and tail length grew bigger and longer in the older juveniles, the intestine length increased isometrically with height. Ingested live food was poorly digested in early juveniles and some Artemia nauplii were found to be still alive in the excreta.

Interpretation: Overall, the results indicated that the early feeding of $H$. kuda was highly inefficient. Depletion of remnant yolk in $H$. kuda suggested partial lecithotrophy during the first two days. These findings provide an important insight for developing nursing methods for this seahorse species.

Key words: Critical period, Digestive tract, Histology, Lecithotrophy, Mass mortality

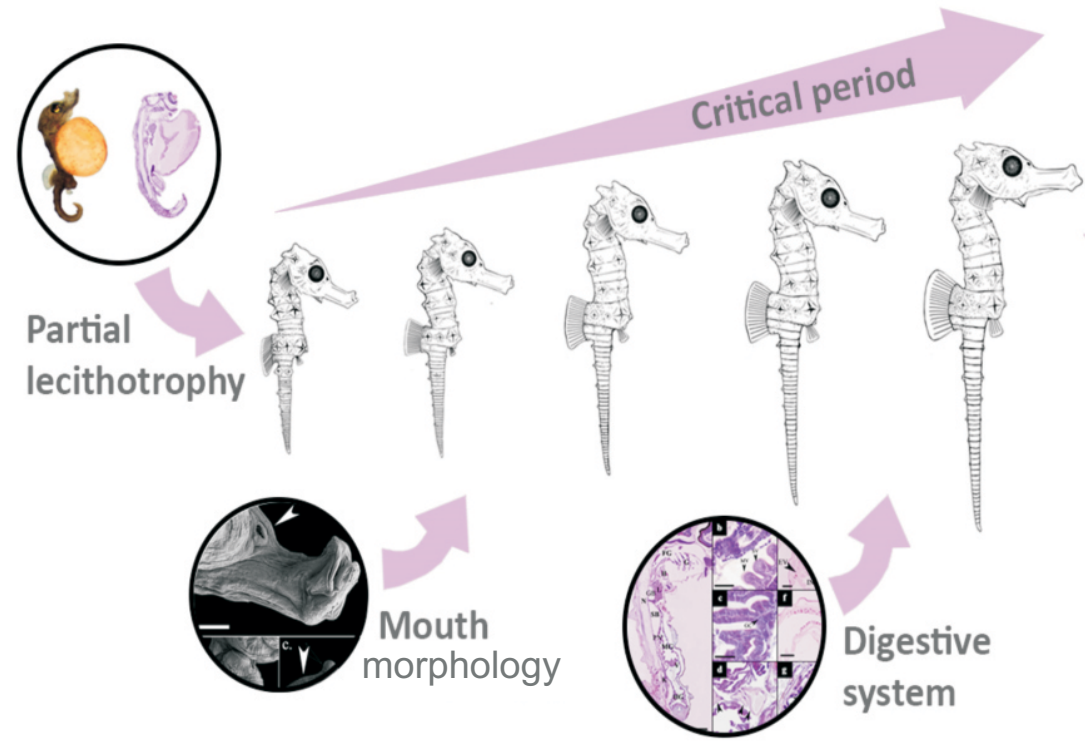

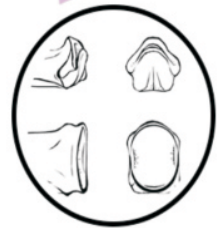

Feeding ability

How to cite : Kamarudin, M.S., N. Romano, A. Arshad, N.L.W.S. Wong and J.C. Teh: External and internal morphology, and feeding ability of yellow seahorse Hippocampus kuda (Bleeker, 1852) early juveniles. J. Environ. Biol., 41, 1138-1146 (2020). 


\section{Introduction}

Seahorses are popular ornamentals due to their adorable and charismatic morphological appearance that includes longsnout, fused-jaws, hard bony-plated body and a prehensile tail (Lourie et al., 2004). However, high mortalities and/or total mortality that often occur in the critical period, shortly after newborns are released from the male's brood pouch are a major setback for aquaculture and conservation management of Hippocampus kuda (Lin et al., 2006; Celino et al., 2012). The phenomenon of mass mortality is also commonly reported in other teleost fish species, which usually starts at larval first exogenous feeding and lasts for 7-10 days (Sifa and Mathias 1987; Jobling et al., 2012; Browman, 2014). Some of the reasons that are attributed to the early mass mortality includes nutritional deficiencies that can stem from limited feeding ability, immature digestive system, as well as unsuitable and/or inadequate live foods (Rønnestad et al., 2013; China and Holzman, 2014; Novelli et al., 2016).

Although the embryonic and post-natal development (from birth to 123 days after birth, DAB) of $H$. kuda has been well delineated by Mi et al. (1998) and Choo and Liew (2006). The link between mass mortality and feeding ability of $H$. kuda juveniles during critical period, which is closely associated with their morphological development, has not been addressed in the available reports. In recent years, some studies have focused on the internal morphology of juvenile seahorses (Palma et al., 2014; Novelli et al., 2015; Ofelio et al., 2018). Despite exogenous feeding among newborn seahorses, the presence of yolk internally during early days of juvenile seahorse and internal developments such as formation of intestinal loops in Hippocampus reidi juveniles strongly indicate that early juvenile seahorses have immature digestive system (Novelli et al., 2015).

To the best of our knowledge, no report has been published on the internal morphology or alimentary system of $H$. kuda juveniles. Nevertheless, the purpose of this study was not to describe the ontogeny of digestive system and morphological developments, but only to examine the allometric changes to the feeding and digestive system of $H$. kuda juveniles in the immediate period after birth. This was done to better understand the feeding efficiency of $H$. kuda during this critical period in order to mitigate the problems of mass mortality and improve the culture technique for conservation of this seahorse species.

\section{Materials and Methods}

Source of experimental animals: Pregnant $H$. kuda males were collected from the seagrass meadow at Merambong Shoal, Johor, Malaysia and transported in a 10 I container with air supply to the International Institute of Aquaculture and Aquatic Sciences (I-AQUAS), Universiti Putra Malaysia, Port Dickson, Negeri Sembilan, Malaysia. Juvenile seahorses used for the external and internal morphological studies were derived from two different pregnant males. After giving birth, all adult seahorses were returned to their original habitat.

Seahorse rearing : In all 250 newborn seahorses released from the first pregnant male's brood pouch were collected immediately using a scoop-net and disinfected in a 30-ppm formalin bath for 1 hr (Buen-Ursua et al., 2011). Three apparently normal newborn seahorses were selected, snap-frozen in liquid nitrogen and then kept at $-80^{\circ} \mathrm{C}$. These specimens were processed for scanning electron microscopy. Meanwhile, five apparently premature seahorses and five normal newborn seahorses were selected and fixed in $10 \%$ buffered formalin for $24 \mathrm{hr}$ followed by storage in $70 \%$ ethanol until microscopic inspections within 1 month.

After preliminary assessment based on the descriptive quality that included relatively larger size, darker skin pigmentation and stronger swimming strength (Zhang et al., 2015), 30 healthy newborn seahorses were selected and equally placed into three culture tanks (10 newborns tank ${ }^{-1}$ ) filled with 10 I of filtered $(1-\mu \mathrm{m})$ and UV-sterilized seawater. Gentle aeration was provided and approximately $10^{4}$ cells $\mathrm{ml}^{-1}$ microalgae Nannochloropsis oculata was added to each tank (Mélo et al., 2016). All seahorses were fed a mixture of rotifers Brachionus sp., Artemia nauplii and enriched Artemia metanauplii (Easy DHA Selco, INVE Aquaculture, Thailand) following the method of Teh et al. (2018). Faeces and debris at the bottom of culture tanks were siphoned out and approximately $20 \%$ water exchange were performed twice daily (09:00 and 17:00hr). Water temperature, salinity, pH and dissolved oxygen were measured every 2 day using a multi-parameter meter (YSI ProPlus, YSI Inc., USA) that ranged $27.1 \pm 1.6^{\circ} \mathrm{C}, 30.5 \pm 1.2 \%, 7.8 \pm 0.2$ and $4.2 \pm 0.6 \mathrm{mg} \mathrm{l}^{-1}$, respectively, while ammonia-nitrogen level was tested with a chemical test kit (API, Chalfont, USA) and was found consistently below $0.05 \mathrm{mg} \mathrm{l}^{-1}$. Two juveniles were randomly selected from each tank on 3, 5, 7 and $9 \mathrm{DAB}$, and fixed in $10 \%$ formalin for $24 \mathrm{hr}$ and then stored in $70 \%$ ethanol for microscopic study.

For histological study, five underdeveloped seahorses and ten normal newborn seahorses released from second pregnant male broodstock were fixed in $10 \%$ buffered formalin. After $24 \mathrm{hr}$ fixation, these specimens were decalcified and preserved in $70 \%$ ethanol for histological procedures. In all 200 newborn seahorses were equally placed into two circular tanks (100 newborns tank $\left.{ }^{-1}\right)$ filled with 50 I of filtered $(1-\mu \mathrm{m})$ and UVsterilized seawater and cultured similarly as described above. Five juveniles were randomly selected from each culture tank and were formalin fixed for later histological procedures on 1, 3, 5, 7 and $9 \mathrm{DAB}$. The average wet weight of the seahorses were measured on analytical balance, while the heights were measured using a calliper snapshot with a digital camera (Olympus TG-4). Meanwhile, faeces of seahorses were sampled randomly, about 10-15 min after live food organisms were offered every morning. The faecal sac was collected as soon as it was 
excreted from the anus of seahorse using a $3 \mathrm{ml}$ disposable pipette. The contents of faecal sac were examined immediately under a light microscope. The narrow opening-end of pipette was removed to ensure that the faeces samples were withdrawn smoothly.

Specimen processing for scanning electron microscopy : Samples retrieved from $-80^{\circ} \mathrm{C}$ were fixed in $4 \%$ glutaraldehyde for $24 \mathrm{hr}$ at $4^{\circ} \mathrm{C}$, followed by post fixation in $1 \%$ osmium tetroxide for 2 $\mathrm{hr}$ at $4^{\circ} \mathrm{C}$. After washing with a $0.1 \mathrm{M}$ sodium cacodylate buffer, samples were dehydrated through a series of graded acetone, critical point dried with $\mathrm{CO}_{2}$ and then mounted onto stubs coated with a gold layer using a sputter coater (SCD 005, Baltec, Germany). Samples were viewed with variable pressure scanning electron microscope (LEO 1455, SEMTech Solutions Inc., USA), equipped with energy dispersive spectroscopy system (EDX, Oxford Instruments, UK).

Inspections of external morphology : Seahorse specimens retrieved from $70 \%$ ethanol were patted dried on paper towels before being observed under a light microscope equipped with a LCD camera (Moticam, Motic Group Co. Ltd., China) and associated analytical software (MotiConnect Version 1.1.2). The height, head, snout and tail length were measured with a calliper following the methods of Lourie et al. (2004) and Choo and Liew (2006). The tail length to head length ratio (T:H) and head length to snout length ratio $(\mathrm{H}: \mathrm{S})$ were calculated. The mouth gape size, which is defined as the vertical distance of snout at the narrowest point (Celino et al., 2012), was measured. Considering the opened mouth gape as an ellipsoid, the mouth area of $H$. kuda juveniles was also estimated by the formula: $A=a b \pi$, where, $A$ is the mouth area; $a$ is the semi-vertical axis of open mouth; $b$ is the semi-horizontal axis of open mouth (Blanco, 2014).

Histological analysis : After $24 \mathrm{hr}$, formalin fixed seahorse specimens were rinsed with deionized distilled water, patted dried and decalcified (Decalcifying Solution-Lite D 0818, SigmaAldrich, Inc.) before the routine processing for paraffin embedment and histological sections (LePage et al., 2015). Sagittal sections measuring 5.0-6.0 $\mu \mathrm{m}$ thickness were cut with a rotary microtome (Microm HM 340E, Thermo Scientific). Sections were stained with haematoxylin and eosin and periodic acidSchiff (PAS), and then mouted with DPX. All slides were examined and photographed under a compound microscope (Olympus BX43F) with a digital camera (Olympus DP22) linked to a computer with image-processing software (Olympus cellSens Standard V1.14). The length of intestine was measured (proximate length from the beginning of foregut to the end of hindgut) while the intestine length to height ratio (IL:H) was calculated (Yip etal., 2015).

\section{Results and Discussion}

Premature seahorses possessed a round-shaped yolk sac that was attached to their bodies (Fig. 1), but this feature was not observed in normal newborn seahorses. The premature seahorse had smooth body, fins, eyes, opened mouth and closed anus (Fig. 1a). The intestine of a premature newborn was not fully differentiated and was open to the yolk sac when its histology sections were investigated under a microscope (Fig. 1b).

Normal newborn seahorses had a dark skin colour with heavy pigmentation. Nares were observed using SEM (Fig. 2a), but not under the light microscope. The details of the mouth morphology and structure were observed (Fig. 2a). The prominent hard bony plated body in the newborn (Fig. 2b) became smoother by $9 \mathrm{DAB}$, but the blunt coronet (Fig. 2c) became sharper and protruded. The head was larger but the tail was shorter in a newborn compared to the older seahorses (Fig. 3). This developmental trait was further validated by increased T:H ratios in the developing $H$. kuda juveniles from 1.3 to 2.0 (Table 1).

Meanwhile, the $\mathrm{H}: \mathrm{S}$ ratio in juveniles during the first 10 days remained similar at 2.2, which reconfirmed that the allometric growth of head and snout in $\mathrm{H}$. kuda juveniles were isometric (Table 1). Both mouth gape size and mouth area increased from 0.26 to $0.53 \mathrm{~mm}$ and from 0.070 to $0.165 \mathrm{~mm}^{2}$, respectively, as $\mathrm{H}$. kuda juveniles developed (Table 1). The mouth morphology of $H$. kuda juveniles is illustrated in Fig. 4. The height, tail length, head length and snout length of $H$. kuda juvenile increased at different allometric growth rates during first 10 days (Fig. 5). The tail showed the highest growth rate of $0.322 \mathrm{~mm} \mathrm{~d}^{-1}$, followed by head $\left(0.074 \mathrm{~mm} \mathrm{~d}^{-1}\right)$ and snout $\left(0.036 \mathrm{~mm} \mathrm{~d}^{-1}\right)$.

A normal newborn $\mathrm{H}$. kuda had a tubular snout, straight intestinal tube with open anus, as well as gallbladder, liver and pancreas (Fig. 6a). The mean weight, height and length of the intestine increased with the age of seahorses, however IL:H ratios remained the same $(0.30)$ (Table 2 ) and no loop formation was observed during the first 10 days. The alimentary tract could be differentiated by an oesophagus at the foregut, anterior intestine at the midgut, posterior intestine at the hindgut, and an ileocecal valve was present between the midgut and hindgut (Fig. 6a). The midgut and hindgut were similar in structure during 0 to 5 $\mathrm{DAB}$, and were characterized by a spacious lumen and monostratified epithelial cells with apical microvilli (Fig. 6b). The whole gut epithelium was brush-bordered. Acidophilic yolk globules were detected near the tubular intestine of newborn seahorses but these globules were absent in juveniles after 1 DAB (Fig. 6c). These globules were likely to be the residues of yolk. An extensive network of longitudinal mucosal folds was observed in the posterior intestine of juvenile seahorses from 7 to $9 \mathrm{DAB}$ (Fig. 6d). Zymogen granules were observed in the pancreatic tissue while glycogens were predominantly located in the liver (Fig. 6e) as well as mucous cells throughout the oesophagus (Fig. 6f). Some basophilic oval bodies were observed to protrude at the lower pharynx, which could be the taste buds (Fig. 6g). The number of mucous cells and supranuclear bodies that were observed in 
(a)

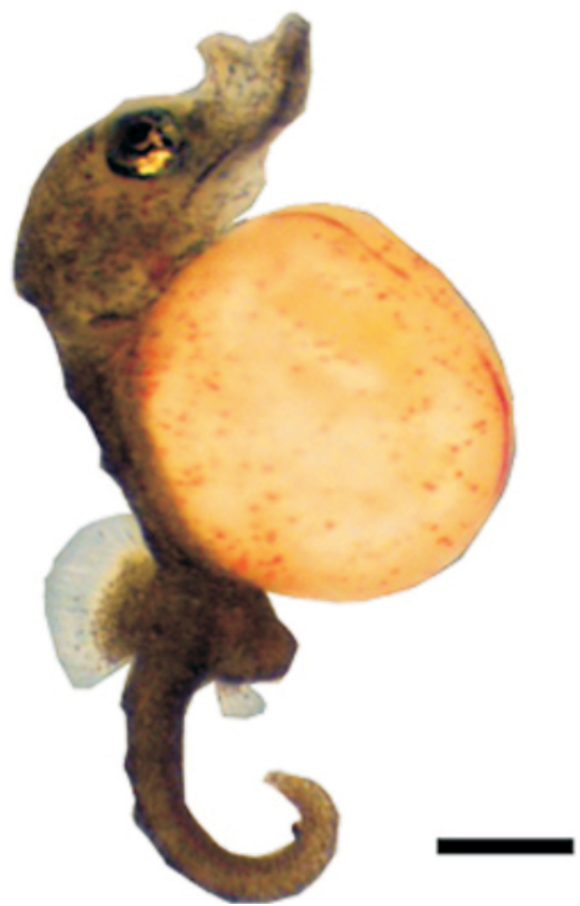

(b)

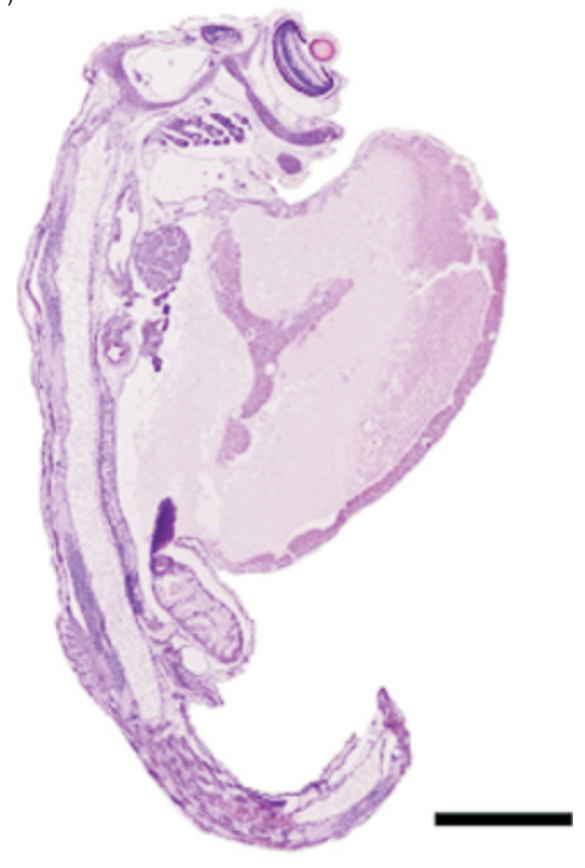

Fig. 1 : Premature seahorse Hippocampus kuda (a) with a round-shaped yolk sac attached to the body; (b) Sagittal section of a premature newborn (H\&E); Scale bar: $500 \mu \mathrm{m}$.
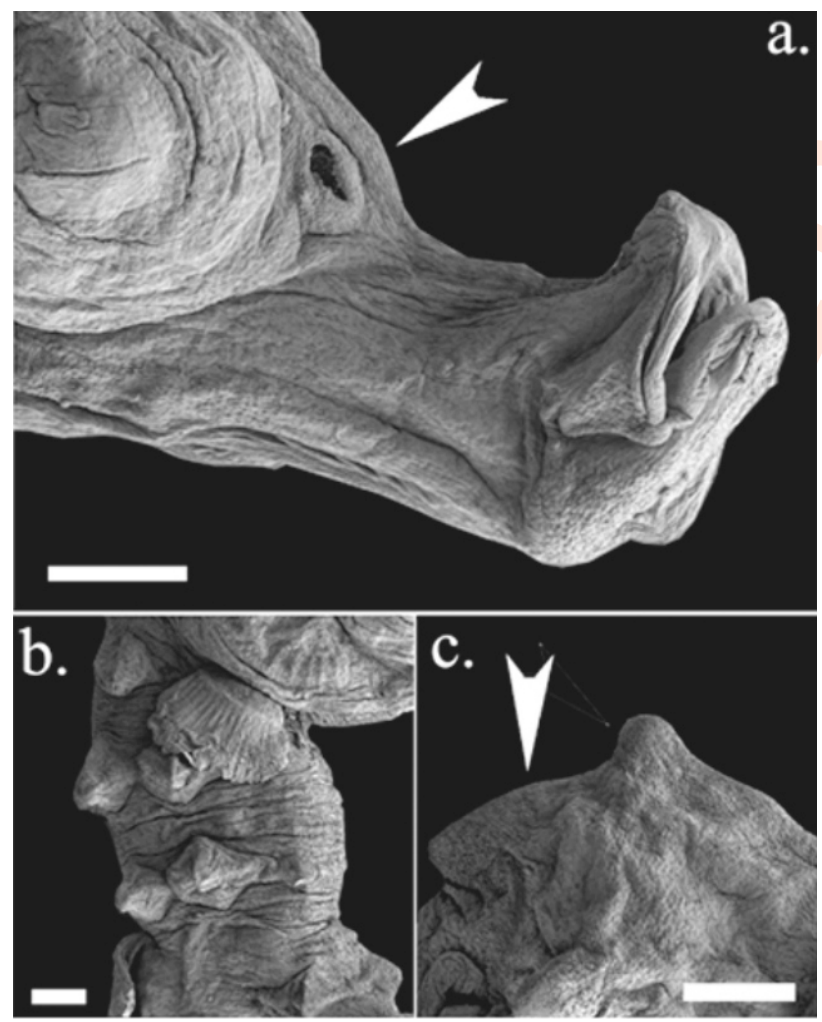

Fig. 2 : Scanning electron monographs (SEM) of newborn Hippocampus kuda, (a) nares and the mouth morphology, (b) tubercles on the hard bony-plated trunk and (c) the blunt coronet were observed. Scale bars: $200 \mu \mathrm{m}$. midgut and hindgut increased with seahorse development (Fig. $6 b, d)$.

Prey organisms were captured and swallowed as whole by juvenile seahorses. Evacuated faeces observed under a light microscope showed that ingested live foods were wrapped in mucous substances (Fig. 10) and some of the encapsulated Artemia nauplii were still alive in the excreta during 0-2 DAB.

Seahorses have high parental investment as the growth and development of seahorse embryos and larvae are carried out in the brood pouch of males (Mi et al., 1998). Such reproduction strategy is very different from most marine fishes, which spawn and fertilize in water, and the young undertake dramatic metamorphosis with multiple different larval and juvenile stages (Osse et al., 1997; Rønnestad et al., 2013). Nevertheless, newborn seahorses are still not really competent when first released and often die en masse (Lin et al., 2006; Otero-Ferrer et al., 2010; Celino et al., 2012). Although the morphological transition observed in $H$. kuda early juveniles was not metamorphosis, this phase of early post-release development was critical to survival of the individual and the brood. Results and observations in this study indicated that the major bottleneck for survival of early seahorses may be the efficiency of digestive system, which took more than 10 days to develop after birth. Thus, a high level of nursery care during early stage could be the most viable approach for nursing and conservation of $H$. kuda. 
Table 1 : Morphological characteristics of Hippocampus kuda from 0 to 9 day-after-birth (DAB).

\begin{tabular}{llllll}
\hline Morphological characters & DDAB & 3DAB & 5DAB & 7DAB & 9 DAB \\
\hline${ }^{*} \mathrm{~T}: \mathrm{H}$ & $1.2-1.3(1.3)$ & $1.3-1.4(1.4)$ & $1.6-1.7(1.7)$ & $1.8-1.9(1.8)$ & $2.0-2.1(2.0)$ \\
${ }^{*} \mathrm{H}: \mathrm{S}$ & $2.2-2.3(2.2)$ & $2.2(2.2)$ & $2.1-2.3(2.2)$ & $2.2(2.2)$ & $2.2-2.3(2.2)$ \\
Trunk rings & 11 & 11 & 11 & 11 & 11 \\
Tail rings & 36 & 36 & 36 & 36 & 36 \\
Dorsal fin rays & 17 & 17 & 17 & 17 & 17 \\
Pectoral fin rays & 16 & 46 & 16 & 4 & 16 \\
Anal fin rays & 4 & $0.32 \pm 0.03$ & $0.38 \pm 0.04$ & $0.45 \pm 0.06$ & $0.53 \pm 0.08$ \\
Mouth gape $(\mathrm{mm})$ & $0.26 \pm 0.03$ & $0.077 \pm 0.005$ & $0.106 \pm 0.013$ & $0.135 \pm 0.018$ & $0.165 \pm 0.025$ \\
Mouth area $(\mathrm{mm} 2)$ & $0.070 \pm 0.004$ & &
\end{tabular}

$\mathrm{T}: \mathrm{H}$ is the ratio of tail length to head length; $\mathrm{H}: \mathrm{S}$ is the ratio of head length to snout length; and meristic counts in present study are given as modal counts; *data presented as minimum, maximum and mode (in parentheses), $n=6$.

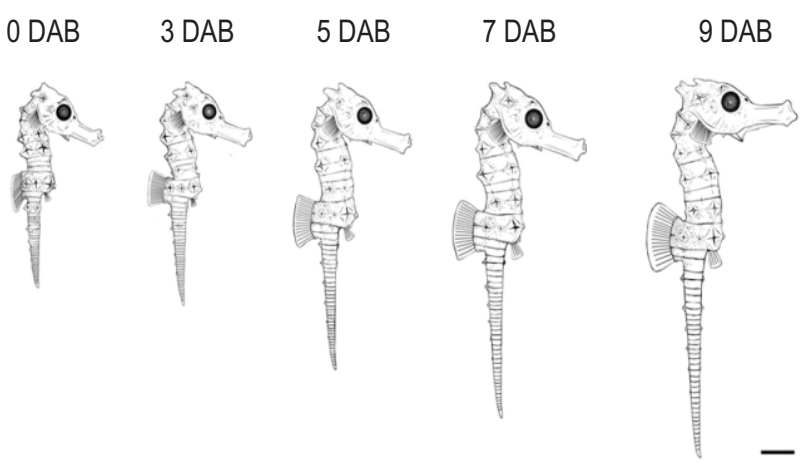

Fig. 3 : Morphological development of Hippocampus kuda juvenile during the first 10 days. Scale bar: $1 \mathrm{~mm}$.

It was observed that $1-3 \%$ of newborn seahorses were not fully matured and carried a spherical yolk sac externally. Premature seahorses were observed to recumbent at the bottom of culture tank and their body attached with yolk sac was soft and vulnerable to predators. Although there was no exogenous feeding and had an undifferentiated alimentary system, energy reserves in the yolk might sustain the survival of some premature seahorses up to two days after being released. Premature seahorse larvae are usually released in a single batch or in batches over several days when the male is under stress or shock (Olivotto et al., 2011; Sommer et al., 2012). In the present study, premature seahorse larvae were released almost simultaneously with well-developed juveniles. A similar observation was also reported by Mi et al. (1998) who suggested that seahorses are perhaps brooded at different developmental stages. Similarly, a small percentage of premature or non-viable eggs among newly released Hippocampus abdominalis and Hippocampus guttulatus juveniles have been reported (Woods, 2005; Blanco, 2014).

Compared to the premature seahorse, the normal newborn $H$. kuda had a bony-plated body with tubercles that presumably acted as mechanical protection from predators during pelagic stage. Newborn seahorses are capable of swimming and feeding independently and both their mouth and anus are open (Kornienko, 2001; Sommer et al., 2012). However, acidophilic bodies are found scattered around the gut tube that indicate yolk residue based on similar characteristics reported in fish larvae (Comabella, et al., 2013; Novelli et al., 2015), such as spherical droplets or bubbly structures that are acidophilic. A similar observation was also reported by Novelli et al. (2015) when observing histopathological changes during early ontogenetic development of $H$. reidi juveniles. The internal yolk reserves in $H$. reidi juveniles was reabsorbed and depleted 2 $D A B$, while in this study, the yolk residues in $H$. kuda disappeared by $1 \mathrm{DAB}$. Although food was immediately ingested after birth, the presence of yolk residues might allow for a certain degree of lecithotrophy in juvenile seahorses during the first few days. This feature is common among various species of marine fish larvae during transition from endogenous to exogenous feeding (Jaroszewska and Dabrowski, 2011; Comabella et al., 2013). Newborn $H$. kuda has been reported to survive starvation for 3 days without affecting their first feeding rate while its point-of-noreturn (PNR) is $115.6 \mathrm{hr}$ (Sheng et al., 2007). Meanwhile, high survival among juveniles of different seahorse species were also observed during the first few days, irrespective of their feeding regimes (Otero-Ferrer et al., 2010; Pham and Lin, 2013).

As observed in this study, the pelagic $H$. kuda juveniles had a large mouth gape that could be opened up to $90^{\circ}$ and their snout could be expanded nearly 1.5 times bigger to maximize their ingestion capacity. The mouth area in 9 DAB $H$. kuda was double that of a newborn. Selection of live foods that compatibly match with the mouth morphology and feeding habit is important for the successful feeding in juvenile seahorses (Celino et al., 2012; Souza-Santos et al., 2013). Olfactory openings have been reported in the postembryonic $H$. kuda larvae in the paternal brood pouch (Mi et al., 1998). Similarly, nares observed in this study indicated that newborn $H$. kuda were likely to have a 


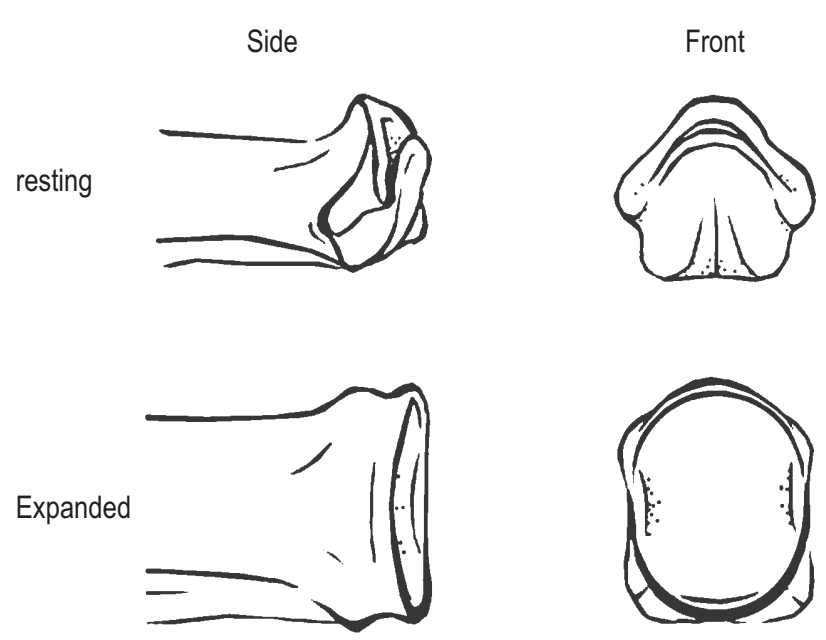

Fig. 4 : Side and front views of the mouth opening and snout expansion of Hippocampus kudajuvenile.

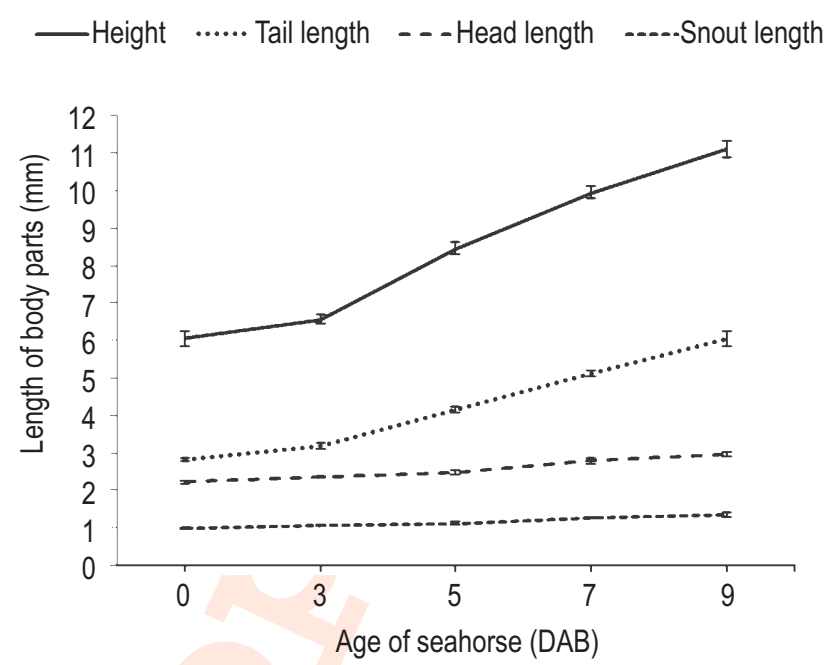

Fig. 5 : Allometric growth of various body parts in Hippocampus kuda juveniles from 0 to 9 day-after-birth (DAB). Values are Mean \pm SD.

Table 2: Weight, height, intestine length and intestine length to height ratio (IL:H) of Hippocampus kuda juveniles from 0 to 9 day-after-birth (DAB).

\begin{tabular}{lllll}
\hline DAB & Wetweight $(\mathrm{mg})$ & Height $(\mathrm{mm})$ & Intestine length $(\mathrm{mm})$ & IL:H \\
\hline 0 & $5.06 \pm 0.04$ & $6.12 \pm 0.28$ & $1.79 \pm 0.06$ & 0.29 \\
1 & $5.36 \pm 0.02$ & $6.27 \pm 0.52$ & $1.86 \pm 0.06$ & 0.30 \\
3 & $5.39 \pm 0.34$ & $7.79 \pm 0.39$ & $2.33 \pm 0.25$ & 0.30 \\
5 & $6.97 \pm 0.33$ & $10.25 \pm 0.50$ & $3.02 \pm 0.19$ & 0.29 \\
7 & $8.32 \pm 0.31$ & $11.62 \pm 0.65$ & $3.46 \pm 0.26$ & 0.30 \\
9 & $10.02 \pm 0.35$ & $12.81 \pm 0.77$ & $3.89 \pm 0.40$ & 0.30 \\
\hline
\end{tabular}

Wet weight, height and intestine length data are presented as mean of ten replicates \pm S.D.

functional olfactory sensory organ and this could be a decisive organ for juvenile seahorses to successfully locate and capture live prey. Other than food searching, olfaction is also important for avoiding predators and finding a mate (Bahr et al., 2012; Lin et al., 2016). Lin et al., 2016 revealed that $H$. comes has a reduced number of olfactory receptor (OR) genes compared to other teleost fish, and all of the identified OR genes are only for detecting water-soluble odorants.

Seahorses have a full complement of rings and fin rays upon birth (Lourie et al., 2004; Choo and Liew, 2006). It is common that the priority of biological development is given initially to the vital organs, especially head for enhanced survival in fish larvae (Osse et al., 1997; Choo and Liew, 2006). In contrast, the tail of $H$. kuda juvenile did not seem to have any particular or vital function during the first 10 days. The allometric growth of tail thereby could be linked to an improved nutritional utilization and allocation in $\mathrm{H}$. kuda juveniles. Indeed, newborn $\mathrm{H}$. kuda had a relatively larger head but shorter tail compared to older juveniles, and these were validated by increased T:H ratio from 1.3 to 2.0 during the first 10 days. A higher $\mathrm{T}: \mathrm{H}$ ratio could perhaps indicate a better foraging capability as well as better health status among the developing $H$. kuda juveniles. Meanwhile, the $H: S$ ratio was 2.2 throughout first 10 days. Since the $\mathrm{H}: \mathrm{S}$ ratio in this study was also similar to that reported in $\mathrm{H}$. kuda adults (Lourie et al., 2004; Choo and Liew, 2006), it reaffirms the ratio as being species-specific. These morphometric data could aid in the conservation management and identification of wild drifting seahorse species at juvenile stages (Vincent et al., 2011; Luzzatto et al., 2013; Wong and Teh, 2017).

Histological analysis revealed that $H$. kuda juveniles were stomachless and possessed no teeth or any internal masticatory structures that would be necessary to mechanically break down ingested food. Taste buds observed at pharynx indicated that food selectivity and foraging behaviour of early $\mathrm{H}$. kuda juveniles could be determined by their gustatory sense as well (Comabella et al., 2013; Novelli et al., 2015). Neutral mucosubstances and mucosal cells were heavily stained with Periodic-acid Schiff, especially in the oesophagus and midgut, indicating high production of mucous in these areas. Mucous is produced to presumably facilitate peristalsis movement of food substances throughout the alimentary tract and provide immunological barriers against microorganisms (Leknes, 2011). Similar to this study, live foods 


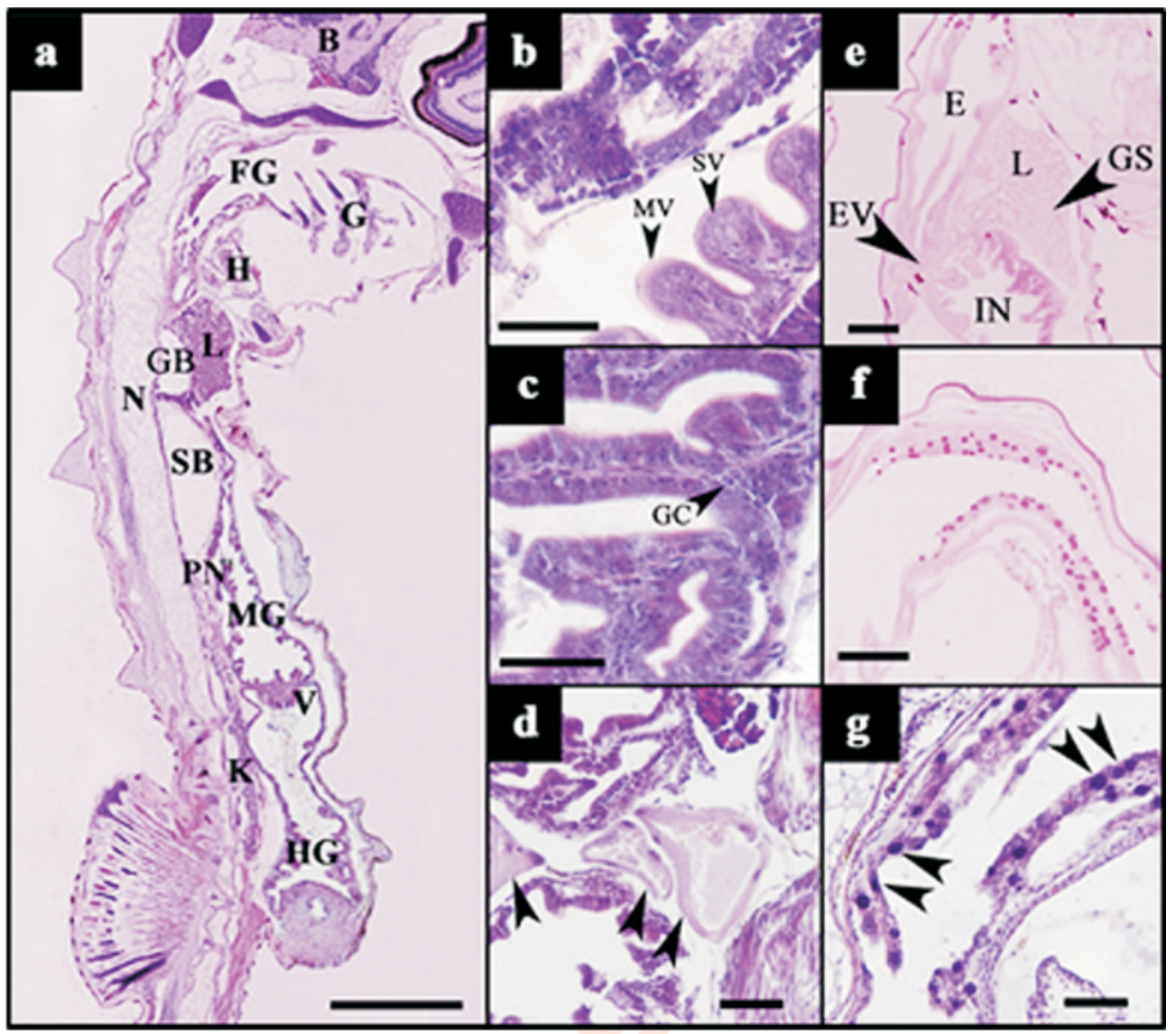

Fig. 6 : Sagittal sections of Hippocampus kuda juveniles. (a) Internal morphology and alimentary tract of 0-9 DAB juveniles (H\&E, scale bar: $500 \mu \mathrm{m})$. (b) Epithelial cells with apical microvilli and supranuclear vesicles (H\&E, scale bar: $40 \mu \mathrm{m}$ ). (c) Longitudinal folds at the hindgut of 7-9 DAB juveniles (H\&E, scale bar: $40 \mu \mathrm{m}$ ). (d) Residues of yolk sac scattered around the intestinal tube at 0 DAB (H\&E, scale bar: $50 \mu \mathrm{m}$ ). (e) Glycogen storage in the liver of 7 DAB seahorse (PAS, scale bar: $100 \mu \mathrm{m}$ ). (f) Mucous cells in oesophagus of 3 DAB seahorse (PAS, scale bar: $100 \mu \mathrm{m})$. (g) Taste buds at the pharynx (H\&E, scale bar $50 \mu \mathrm{m})$. B, brain; E, oesophagus; EV, valve between oesophagus and intestine; FG, foregut; G, gills; GB, gallbladder; GC, goblet cell; GS, glycogen storage; H, heart; HG, hindgut; IN, intestine; K, kidney; L, liver; MG, midgut; MV, microvilli; N, notochord; PN, pancreas; SB, swim bladder; SV, supranuclear vesicles; $\mathrm{V}$, valve between midgut and hindgut.

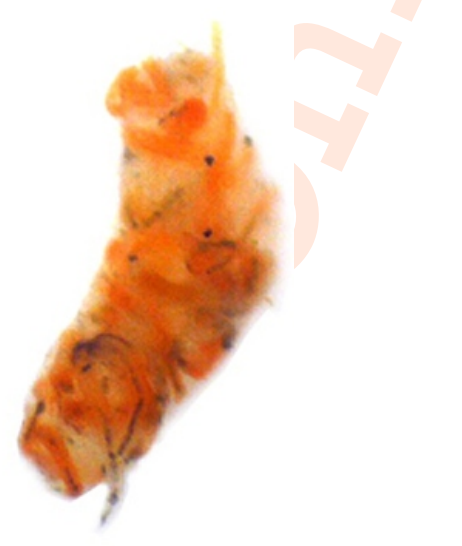

Fig. 7 : Faeces evacuated from Hippocampus kuda juveniles. Food organisms were wrapped in mucous substances. Scale bar: $1 \mathrm{~mm}$. are also wholly eaten by the juveniles of other seahorse species and some often remain structurally intact and wrapped in mucous substances or peritrophic-like viscous sac when evacuated in the faeces (Córdova-Murueta et al., 2003; Blanco et al., 2016). Moreover, the presence of some live but encapsulated Artemia nauplii in the faeces have also been reported in $\mathrm{H}$. abdominalis and $H$. guttulatus juveniles (Woods, 2003; Blanco et al., 2016).

This phenomenon of live excreted nauplii could be due to combination of several reasons. For instance, Blanco et al. (2016) speculated that this might be due to the immature digestive system of juvenile seahorses. In addition, the absence of internal structures necessary for mastication could also contribute to this phenomenon since autolysis of live food only starts when food organisms die in the fish gut. Another possible contributor could be the short and consistent relative intestine length $(\mathrm{IL}: \mathrm{H})$ of early 
H. kuda juveniles from 0 to $9 \mathrm{DAB}$ observed in the current study. Thus, this would likely lead to a shorter gut evacuation time as a longer time is necessary for enzymes to digest live foods (Kramer and Bryant, 1995; Rønnestad et al., 2013). Higher IL:H ratios of 0.32-0.44 had been reported in the older seahorses Hippocampus spinosissimus and Hippocampus trimaculatus (Yip et al., 2015) and this is largely due to the formation of intestinal loops which would greatly increase the food passage time and surface area. The first intestinal loop is formed 12 and 15 DAB in H. reidi and H. guttulatus (Novelli et al., 2015; Ofelio et al., 2018). In this study, however, no loop was observed during the first 10 days of $H$. kuda species.

This study revealed that early juveniles of Hippocampus kuda (Bleeker, 1852) underwent a series of external and internal morphological developments, which likely resulted in poor digestion of certain live food and mass mortality during the early stage. Some essential knowledge for conservation management and developing nursery techniques for this seahorse species were discussed. Further investigations on the digestive chemistry of $H$. kuda juveniles might provide greater insights into their food digestion and digestive capacity.

\section{Acknowledgments}

This study was funded by a grant from Universiti Putra Malaysia (GP-IPB/2014/9440402). The authors are grateful to Kelab Alami Tanjung Kupang for their assists in field work.

\section{References}

Bahr, A., S. Sommer, B. Mattle and B. Wilson: Mutual mate choice in the potbellied seahorse (Hippocampus abdominalis). Behav. Ecol., 23, 869-878 (2012).

Blanco, A., M. Planas and J. Moyano: Ontogeny of digestive enzymatic capacities in juvenile seahorses Hippocampus guttulatus fed on different live diets. Aquac. Res., 47, 3558-3569 (2016).

Browman, H.I.: Commemorating 100 years since Hjort's 1914 treatise on fluctuations in the great fisheries of northern Europe: Where we have been, where we are, and where we are going. ICES J. Mar. Sci., 71, 1989-1992 (2014).

Buen-Ursua, S.M.A., T. Azuma, C.P. Recente and R.E. Batatin: Effects of UV-treated sea water, chlorinated sea water, and formalin-treated copepods on survival and growth of newborn seahorses, Hippocampus comes. Isr. J. Aquacult. Bamidgeh, 63, 629-635 (2011).

Celino, F.T., G.V. Hilomen-Garcia and A.G.C. Norte-Campos: Feeding selectivity of the seahorse, Hippocampus kuda (Bleeker), juveniles under laboratory conditions. Aquacult. Res., 43, 18041815 (2012).

China, V. and R. Holzman: Hydrodynamic starvation in first-feeding larval fishes. Proc. Natl.Acad. Sci. U.S.A., 111, 8083-8088 (2014).

Choo, C.K. and H.C. Liew: Morphological development and allometric growth patterns in the juvenile seahorse Hippocampus kuda Bleeker. J. Fish Biol., 69, 426-445 (2006).

Comabella, Y., A.H. Franyutti, A. Hurtado, J. Canabal and T. GarciaGalano: Ontogenetic development of the digestive tract in Cuban gar (Atractosteus tristoechus) larvae. Rev. Fish Biol. Fisher., 23, 245-260 (2013).

Córdova-Murueta, J.H., F.L. Garcia-Carreno and M.A. Navarrete-delToro: Digestive enzymes present in crustacean feces as a tool for biochemical, physiological, and ecological studies. J. Exp. Mar. Biol. Ecol., 297, 43-56 (2003).

Jaroszewska, M. and K. Dabrowski: Utilization of yolk: Transition from endogenous to exogenous nutrition in fish. In: Larval Fish Nutrition (Ed.: G.J. Holt). John Wiley \& Sons, Inc., Oxford, pp. 183-218 (2011).

Jobling, M., A. Alanärä, S. Kadri and F. Huntingford: Feeding biology and foraging. In: Aquaculture and Behavior (Eds.: F. Hungtingford, M. Jobling and S. Kadri). Blackwell Publishing Ltd., Oxford, pp. 121$149(2012)$

Kornienko, E.S.: Reproduction and development in some genera of pipefish and seahorses of the family Syngnathidae. Russ. J. Mar. Biol., 27, S15-S26 (2001).

Kramer, D.L. and M.J. Bryant: Intestine length in the fishes of a tropical stream: 2. Relationships to diet - the long and short of a convoluted issue. Environ. Biol. Fish., 42, 129-141 (1995).

Leknes, I.L.: Histochemical studies on mucin-rich cells in the digestive tract of a teleost, the Buenos Aires tetra (Hyphessobrycon anisitsi). Acta Histochemica, 113, 353-357 (2011).

LePage, V., J. Young, C.J. Dutton, G. Crawshaw, J.A. Pare, M. Kummrow, D.J. McLelland, P. Huber, K. Young, S. Russell, L. AlHussinee and J.S. Lumsden: Diseases of captive yellow seahorse Hippocampus kuda Bleeker, pot-bellied seahorse Hippocampus abdominalis Lesson and weedy seadragon Phyllopteryx taeniolatus Lacepede. J. Fish Dis., 38, 439-450 (2015).

Lin, Q., S. Fan, Y. Zhang, M. Xu, H. Zhang, Y. Yang, A.P. Lee, J.M. Woltering, V. Ravi, H.M. Gunter, W. Luo, Z. Gao, Z.W. Lim, G. Qin, R.F. Schneider, X. Wang, P. Xiong, G. Li, K. Wang, J. Min, C. Zhang, Y. Qu, J. Bai, W. He, C. Bian, X. Zhang, D. Shan, H. Qu, Y. Sun, Q. Gao, L. Huang, Q. Shi., A. Meyer and B. Venkatesh: The seahorse genome and the evolution of its specialized morphology. Nature, 540, 395-399 (2016).

Lin, Q., J. Lu, Y. Gao, L. Shen, J. Cai and J. Luo: The effect of temperature on gonad, embryonic development and survival rate of juvenile seahorses, Hippocampus kuda Bleeker. Aquaculture, 254, 701$713(2006)$

Lourie, A., S.J. Foster, E.W.T. Cooper and A.C.J. Vincent: A Guide to the Identification of Seahorses. University of British Columbia and World Wildlife Fund, Washington (2004).

Luzzatto, D.C., M.L. Estalles and J.M.D. Astarloa: Rafting seahorses: the presence of juvenile Hippocampus patagonicus in floating debris. J. Fish Biol., 83, 677-681 (2013).

Mélo, R.C.S., L.P. Souza-Santos, A.P.M. Brito, A. Andrade Gouveia, C. Marçal and R.O. Cavalli: Use of the microalgae Nannochloropsis occulata in the rearing of newborn longsnout seahorse Hippocampus reidi (Syngnathidae) juveniles. Aquacult. Res., 47, 3934-3941 (2016).

Mi, P.T., E.S. Kornienko and A.L. Drozdov: Embryonic and larval development of seahorse Hippocampus kuda. Russ. J. Mar. Biol., 24,325-329 (1998).

Novelli, B., F. Otero-Ferrer, M. Diaz, J.A. Socorro, M.J. Caballero, L.M. Dominguez and F.J. Moyano: Digestive biochemistry as indicator of the nutritional status during early development of the long snouted seahorse (Hippocampus reidi). Aquaculture, 464, 196$204(2016)$ 
Novelli, B., J.A. Socorro, M.J. Caballero, F. Otero-Ferrer, A. SegadeBotella and L.M. Dominguez: Development of seahorse (Hippocampus reidi, Ginsburg 1933): Histological and histochemical study. Fish Physiol. Biochem., 41, 1233-1251 (2015).

Ofelio, C., A.O. Diaz, G. Radaelli and M. Planas: Histological development of the long-snouted seahorse Hippocampus guttulatus during ontogeny. J. Fish Biol., 93, 72-87 (2018).

Olivotto, I., M. Planas, N. Simões, G.J. Holt, M.A. Avella and R. Calado: Advanced in breeding and rearing marine ornamentals. J. World Aquacult. Soc., 42, 135-166 (2011).

Osse, J.W.M, J.G.M. Boogaart, G.M.J Snik and L. Sluys: Priorities during early growth of fish larvae. Aquaculture, 155, 249-258 (1997)

Otero-Ferrer, F., L. Molina, J. Socorro, R. Herrera, H. FernándezPalacios and M.S. Izquierdo: Live prey first feeding regimes for short-snouted seahorse Hippocampus hippocampus (Linnaeus, 1758) juveniles. Aquacult. Res., 41, e8-e19 (2010).

Palma, J., D.P. Bureau, and J.P. Andrade: The effect of diet on ontogenic development of the digestive tract in juvenile reared long snout seahorse Hippocampus guttulatus. Fish. Physiol. Biochem., 40, 739-750 (2014).

Pham, N.K. and J. Lin: The effects of different feed enrichments on survivorship and growth of early juvenile longsnout seahorse, Hippocampus reidi. J. WorldAquacult. Soc., 44, 435-446 (2013).

Rønnestad, I., M. Yufera, B. Ueberschar, L. Ribeiro, O. Saele and C. Boglione: Feeding behaviour and digestive physiology in larval fish: current knowledge, gaps and bottlenecks in research. Rev. Aquac., 5, S59-S98 (2013).

Sheng, J., Q. Lin, Q. Chen, L. Shen and J. Lu: Effect of starvation on the initiation of feeding, growth and survival rate of juvenile seahorses, Hippocampus trimaculatus leach and Hippocampus kuda Bleeker. Aquaculture, 271, 469-478 (2007).

Sifa, L. and J.A. Mathias: The critical period of high mortality of larvae fish
- A discussion based on current research. Chinese J. Oceanol. Limnol., 5, 80-96 (1987).

Sommer, S., C.M. Whittington and A.B. Wilson: Standardised classification of pre-release development in male-brooding pipefish, seahorses, and seadragons (Family Syngnathidae). BMC Dev. Biol., 12, 1 (2012).

Souza-Santos, L.P., C.G. Regis, R.C.S. Mélo and R.O. Cavalli: Prey selection of juvenile seahorse Hippocampus reidi. Aquaculture, 404-405, 35-40 (2013).

The, J.C., M.S. Kamarudin, N. Romano, A. Arshad and N.L.W.S. Wong: Dietary effects of rotifers, brine shrimps and cultured copepods on survival and growth of newborn seahorse Hippocampus kuda (Bleeker 1852). J. Environ. Biol., 39, 923-930 (2018).

Vincent, A.C.J., S.J. Foster and H.J. Koldewey: Conservation and management of seahorses and other syngnathidae. J. Fish Biol., 78, 1681-1724 (2011).

Wong, N.L.W.S. and J.C. Teh: Direct observation of drifting juvenile seahorse Hippocampus trimaculatus (Leach, 1814) beneath the offshore surface at Malacca Strait. Check List, 13, 993-996 (2017).

Woods C.M.C.: Effects of varying Artemia enrichment on growth and survival of juvenile seahorses, Hippocampus abdominalis. Aquaculture, 220,537-548 (2003).

Woods C.M.C.: Reproductive output of male seahorses Hippocampus abdominalis, from Wellington Harbour, New Zealand: Implications for conservation. New Zeal. J. Mar. Fresh, 39, 881-888 (2005).

Yip, M.Y., A.C.O. Lim, V.C. Chong, J.M. Lawson and S.J. Foster: Food and feeding habits of the seahorses Hippocampus spinosissimus and Hippocampus trimaculatus (Malaysia). J. Mar. Biol. Assoc. U. K., 95, 1033-1040 (2015).

Zhang, Y., G. Qin, J. Lin and Q. Lin: Growth, survivorship, air-bubble disease, and attachment of feeble juvenile seahorses, Hippocampus kuda (Bleeker, 1852). J. World Aquacult. Soc., 46, 292-300 (2015). 